\title{
$\mathrm{NaCl}$ 胁迫对阿月浑子实生苗活性氧代谢与 细胞膜稳定性的影响
}

\author{
袁 琳 $^{1}$ 克热木・伊力 ${ }^{2}$ 张利权 $1^{*}$ \\ （1 华东师范大学河口海岸国家重点实验室, 上海 200062）（2 新疆农业大学园艺学院, 乌鲁木齐 830052）
}

\begin{abstract}
摘 要 为研究阿月浑子 ( Pistacia vera) 的耐盐性, 对新疆两个主栽品种“长果”和 “Kerman’ 的 1 年生实生苗进行了 控制条件下的 $\mathrm{NaCl}$ 胁迫实验, 实验浓度为 $50 、 150 、 250$ 和 $500 \mathrm{mmol} \cdot \mathrm{L}^{-1}, \mathrm{NaCl}$ 胁迫 $5 、 10$ 和 $20 \mathrm{~d}$ 后取叶片测定其细胞 膜透性、丙二醛 (MDA) 含量以及超氧化物歧化酶 ( SOD) 、过氧化物酶 (POD) 和过氧化氢酶 (CAT) 活性的变化。实验 结果显示, 在 $\mathrm{NaCl}$ 胁迫下, “长果”和 ‘Kerman’ 的膜透性和 $\mathrm{MDA}$ 含量均随 $\mathrm{NaCl}$ 浓度的升高而增加, 表明 $\mathrm{NaCl}$ 胁迫致 使阿月浑子膜脂过氧化程度加强, 细胞膜稳定性受到破坏, 其中 “长果”品种的膜透性和 MDA 含量增加幅度较大, 受到的盐害较大。而 SOD、CAT 和 POD 活性则随 $\mathrm{NaCl}$ 浓度的升高先增加后下降, 抗氧化酶活性 (SOD、POD 和 CAT) 之间协调变化有利于清除活性氧, 维持活性氧代谢平衡, 保护膜结构。实验结果也显示, 随着 $\mathrm{NaCl}$ 胁迫时间的延 长, 两个品种的细胞膜结构和功能受损害程度有所缓解。这些指标中, “长果”和 “Kerman’ 品种的膜透性和 MDA 含 量与 SOD 活性呈显著相关, 表明植物细胞的质膜透性与脂质过氧化产物 (MDA) 含量有关, 也与组织中自由基含量 和保护酶活性密切相关。综合各项生理指标, ‘Kerman’ 品种相对 “长果”品种显示了较强的抗氧化能力, 具有较强的 耐盐能力。
\end{abstract}

关键词 $\mathrm{NaCl}$ 胁迫 阿月浑子 膜透性 丙二醛 超氧化物歧化酶 过氧化物酶 过氧化氢酶

\section{EFFECTS OF NACL STRESS ON ACTIVE OXYGEN METABOLISM AND MEMBRANE STABILITY IN PISTACIA VERA SEEDLINGS}

\author{
YUAN Lin ${ }^{1}$ KARIM Ali ${ }^{2}$ and ZHANG Li-Quan ${ }^{1 *}$ \\ (1 State Key Laboratory of Estuarine and Coastal Research, East China Normal University, Shanghai 200062, China) \\ (2 Institute of Horticulture, Xinjiang Agricultural University, Ürümqi 830052, China)
}

\begin{abstract}
Pistachio ( Pistacia vera) is one of the most important dried fruit trees in the world and is mainly cultivated in Xinjiang, China. However, soil salinization is one of the main limiting factors for its promising potential of development. In this experiment, the effects of $\mathrm{NaCl}$ stress on physiological and biochemical characteristics were investigated in seedlings of two cultivars of Pistacia vera, 'Changguo' and 'Kerman' . Seedlings were grown in pots and treated with four NaCl concentrations: 50, 150, 250 and $500 \mathrm{mmol} \cdot \mathrm{L}^{-1}$. The membrane permeability, malondialdehyde content (MDA), superoxide dismutase (SOD) activity, catalase ( CAT) activity and peroxidase ( POD) activity in the leaves of these two cultivars were measured and compared after 5, 10 and 20 days of $\mathrm{NaCl}$ treatments.

The experimental results showed that the membrane permeability and MDA content in both cultivars increased considerably with the increasing $\mathrm{NaCl}$ stress, which aggravated the degree of membrane lipid peroxidation and injured the membrane stability. The membrane permeability and MDA content in the 'Changguo' cultivar increased more quickly than the 'Kerman' cultivar and thus experienced greater damage at higher $\mathrm{NaCl}$ concentrations. The activities of SOD, CAT and POD had similar trends for both cultivars, first increasing with $\mathrm{NaCl}$ concentrations up to $250 \mathrm{mmol} \cdot \mathrm{L}^{-1}$ and then decreasing at the highest $\mathrm{NaCl}$ stress of $500 \mathrm{mmol} \cdot \mathrm{L}^{-1}$. The coordinated changes of activities among the antioxidant enzymes of SOD, POD and CAT could scavenge active oxygen and maintain a balance of active oxygen accumulation in cells to protect membrane structure. The results also showed that the damage by $\mathrm{NaCl}$ stress to the membrane structure and function for both cultivars were mitigated considerably 20 days after the treatments were initiated. There were significant correlations among the membrane permeability, MDA content and the activity of SOD in both cultivars, which implied that the membrane permeability in the plant cells had a close relation with MDA content and oxygen free radical
\end{abstract}


content, as well as the activity of antioxidant enzymes. Together, these results indicated that the 'Kerman' cultivar has higher antioxidant levels and greater salt-tolerance than the 'Changguo' cultivar of Pistachio.

Key words NaCl stress, Pistacia vera, Membrane permeability, MDA, SOD, CAT, POD

土壤盐渍化严重影响农业生产和生态环境, 成 为阻碍农牧业快速发展的因素之一。目前, 全世界 的盐渍化土地约占陆地面积的 $1 / 3$, 有 100 多个国家 存在着不同类型的盐碱地(林栖风和李冠一, 2000)。 我国干旱地区由于降水少, 气候干燥和成土母质及 灌溉等原因形成了大面积的盐渍土, 总面积约 130 多万平方公里, 且有逐年增加的趋势(王继和和杨自 辉,2001)。这些盐渍土主要分布于东北、山东、河 北、新疆、甘肃等干旱和半干旱及沿海地区。在人口 不断增加, 耕地日趋减少和淡水资源不足的资源环 境压力下,如何利用和开发我国上亿亩盐渍化土壤, 已成为我国农业生产中十分迫切和重要的任务。

新疆是驰名中外的瓜果之乡, 有许多名、优、特、 稀园艺树种资源。目前新疆现有果树面积 197000 $\mathrm{hm}^{2}$ 左右, 占全区耕地面积的 $9 \%$, 预计到 2015 年将 达到 $667000 \mathrm{hm}^{2}$ 以上。新疆受干旱气候和封闭内 陆盆地的影响, 盐碱地具有面积大, 类型多, 积盐重 等特点。新疆的果树主要分布在塔里木盆地周围的 南疆地区, 当地气候干燥、降水量少、蒸发量高, 主要 靠地下水和雪水进行灌溉, 盐碱面积占全区耕地面 积的 $35 \%$ 以上。

阿月浑子 ( Pistacia vera) 商品名称 “开心果”, 系 漆树科黄连木属多年生木本植物 (中国科学院中国 植物志编辑委员会, 1980), 是世界四大干果之一, 也 是我国主要在新疆栽培的很有发展前途的坚果树 种。其苗木的繁殖可采用播种、嫁接、压条与根蓝 法, 在新疆一般采用播种繁殖。实践证明, 阿月浑子 的实生苗变异小, 能基本保持母本树的优良性状(张 开春, 2000)。近年来, 阿月浑子在南疆地区已有一 些发展。然而, 该地区普遍存在的土壤盐渍化对阿 月浑子的果实品质、产量和结果寿命影响很大, 制约 了果树自然资源开发和优良品种引进。

盐胁迫可使植物体内自由基积累量上升, 产生 大量活性氧化物, 加强膜脂过氧化作用, 膜脂过氧化 产物丙二醛 (MDA) 含量增加, 破坏膜结构, 导致膜透 性增加, 最终对细胞造成损伤甚至导致死亡。但细 胞中存在一系列的保护酶, 如超氧化物歧化酶 (SOD)、过氧化物酶( POD) 和过氧化氢酶( CAT) 等, 可在盐胁迫时增强活性, 加快对活性氧的清除, 具有 维持活性氧代谢平衡, 保护膜结构的功能, 是植物能
以忍耐体内高浓度盐的机理之一(潘瑞炽和董愚得, 1995)。迄今为止, 国内外对阿月浑子耐盐性的研究 还很少, 多数是从形态和生长指标方面对 $\mathrm{NaCl}$ 胁迫 下阿月浑子的耐盐性进行初步研究 (Ashworth et al., 1985; Sepskhah \& Maftoin, 1988; Picchioni \& Miyamota, 1990), 而从植株生理生化角度来研究阿月 浑子耐盐机理的研究至今尚未见报道。

本项研究通过不同的 $\mathrm{NaCl}$ 浓度处理, 研究阿月 浑子的新疆地方品种 “长果”和从美国引进品种 “Kerman’ 的细胞膜透性、MDA 含量、SOD 活性、CAT 活性和 POD 活性对 $\mathrm{NaCl}$ 胁迫的反应, 来分析其耐 盐性, 旨在为选育耐盐性强的阿月浑子品种提供科 学理论依据。

\section{1 材料和方法}

\section{1 材料的培养}

本试验采用 2002 年秋播的新疆地方 ‘长果”品 种和从美国引进的 'Kerman’ 品种实生苗为实验材 料。于 2003 年 4 月, 定植于直径为 $21 \mathrm{~cm}$, 深 $25 \mathrm{~cm}$, 盆底有排水孔并置有托盘的花盆中, 每盆种植 1 株 实验材料, 温室中培养。盆土采用新疆当地营养土 与洗净沙子以 $3: 1$ 比例混匀的土壤, 含盐量约为 $0.002 \%$ 。

\section{$1.2 \mathrm{NaCl}$ 处理与取样}

待实验植株长到 $12 \sim 16$ 片叶时, 选取生长健壮 与无病虫害的植株进行 $\mathrm{NaCl}$ 处理。处理设 4 个 $\mathrm{NaCl}$ 浓度 (分别为 $50 、 150 、 250$ 和 $500 \mathrm{mmol} \cdot \mathrm{L}^{-1}$ ) 和 1 个对照, 每组处理 3 个重复。加盐前控制盆水几天, 以利于加盐后盐分在干燥土壤中迅速扩散。不同浓 度的 $\mathrm{NaCl}$ 溶液 (约 $500 \mathrm{ml}$ ) 一次性均匀浇入盆中, 至 花盆下端小孔有液体流出为止, 对照组则浇入自来 水。并在花盆下面放置一个不透水的塑料小盘, 使 下渗的溶液得以保留。处理后, 每天用自来水冲洗 小盘, 并将冲洗液浇入盆土中, 以确保土壤中 $\mathrm{NaCl}$ 的浓度保持在一个稳定的水平。根据盆土湿度, 每 隔 $3 \mathrm{~d}$ 适当浇水 (约 $300 \mathrm{ml}$ ), 以保持土壤水势的稳 定。 $\mathrm{NaCl}$ 胁迫 5、10 和 $20 \mathrm{~d}$ 后取顶芽 2 3 个叶片下 相同位置的成熟健康的展开叶片, 放入液氮中进行 固定, 随后取出叶片去除叶脉, 剪碎混匀放入 -30 ${ }^{\circ} \mathrm{C}$ 低温冰箱保存。 


\section{3 测定项目与方法}

酶液制备时, 取 $0.5 \mathrm{~g}$ 叶片于预冷的研钵中, 加 入 $5 \mathrm{ml}$ 预冷的 $0.05 \mathrm{mmol} \cdot \mathrm{L}^{-1} \mathrm{PBS}(\mathrm{pH}=7.8$ ) 冰浴研 磨, 然后用 $\mathrm{TGL}^{-1} 6 \mathrm{G}$ 台式高速冷冻离心机, 在 $4{ }^{\circ} \mathrm{C}$ 下 $10000 \mathrm{r}^{\bullet} \mathrm{min}^{-1}$ 离心 $20 \mathrm{~min}$, 取上清液, $4{ }^{\circ} \mathrm{C}$ 下保存 用于 SOD、CAT 和 POD 的活性以及 MDA 含量的测定 (李合生, 2000)。

用DDS211AT 型电导仪按电导法测定膜透性 (朱广廉等, 1990)。SOD 活性按 NBT(氮兰四唑)显 色法测定 (李合生, 2000), CAT 活性按紫外吸收法测 定(李合生, 2000), POD 活性采用愈创木酚法测定 (李合生, 2000)。丙二醛含量的测定按赵氏杰等 (1994)方法测定。SOD、CAT、POD 和 MDA 测定时, 样品的吸光度值均用 751-GD 紫外可见光分光光度 计测定。上述每项指标做 3 次重复样品测定, 取平 均值。

实验数据的处理和相关分析用 Microsoft Excel
2003 和 SPSS 11.0 软件完成。

\section{2 结果与分析}

\section{$2.1 \mathrm{NaCl}$ 胁迫对阿月浑子膜透性和 MDA 含量的影} 响

MDA 含量和细胞膜透性与耐盐性的关系密切, 盐胁迫导致叶片中 MDA 积累和细胞膜透性增大。 本项实验显示, 随着 $\mathrm{NaCl}$ 浓度增加, “长果” 和 ‘ Kerman'品种的膜透性和 MDA 含量均迅速升高, 在 $\mathrm{NaCl}$ 浓度为 $500 \mathrm{mmol} \cdot \mathrm{L}^{-1}$ 时达到最大值 (图 1 ), 表明膜 系统功能受损害程度与 $\mathrm{NaCl}$ 浓度有关。 $\mathrm{NaCl}$ 胁迫 10 和 $20 \mathrm{~d}$ 后的膜透性和 $\mathrm{MDA}$ 含量相对 $\mathrm{NaCl}$ 胁迫 5 $\mathrm{d}$ 的略有下降, 表明随着 $\mathrm{NaCl}$ 胁迫时间的延长, 膜 系统功能受损害程度有所缓解。 $\mathrm{NaCl}$ 胁迫 5 与 $10 \mathrm{~d}$ 时, “长果”品种的膜透性比 “ Kerman’ 品种分别高 $10.7 \%$ 和 $8.4 \%$, 而 $\mathrm{NaCl}$ 胁迫 $20 \mathrm{~d}$ 时, 二者的膜透性 相似。然而, “长果”品种膜透性的上升幅度较大, 当
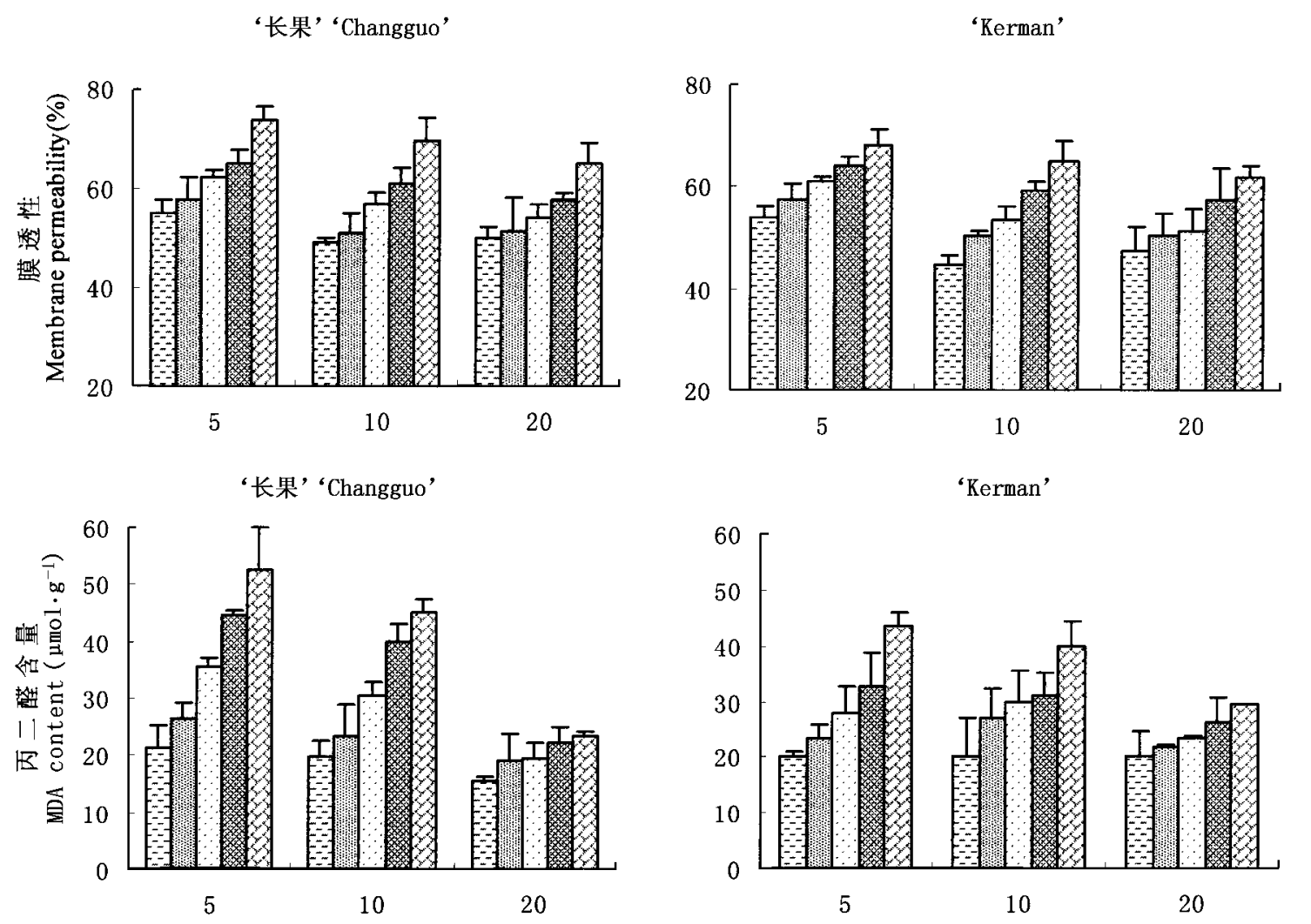

$\mathrm{NaCl}$ 处理天数 $\mathrm{NaCl}$ treatment dâys (d)

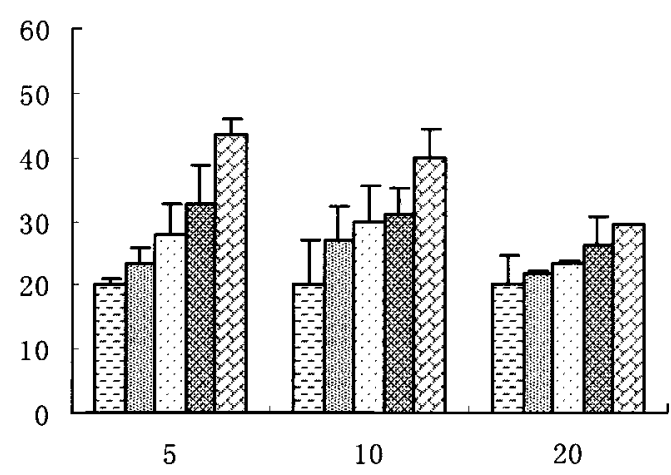

$\mathrm{NaCl}$ 处理天数 $\mathrm{NaCl}$ treatment dâys (d)

口 $\mathrm{CK}$ 图 $50 \mathrm{mmol} \cdot \mathrm{L}^{-1}$ 口 $150 \mathrm{mmol} \cdot \mathrm{L}^{-1}$ 图 $250 \mathrm{mmol} \cdot \mathrm{L}^{-1}$ 日 $500 \mathrm{mmol} \cdot \mathrm{L}^{-1}$ 
$\mathrm{NaCl}$ 浓度从 $250 \mathrm{mmol} \cdot \mathrm{L}^{-1}$ 上升到 $500 \mathrm{mmol} \cdot \mathrm{L}^{-1}$ 时, “长果”品种膜透性的上升幅度比 ‘Kerman’ 品种分别 高 $45.3 \% 、 46.7 \%$ 和 $28.6 \%$ 。 $\mathrm{NaCl}$ 胁迫 5 与 $10 \mathrm{~d}$ 时, ‘Kerman’ 品种的 MDA 含量低于 ‘长果”品种, 而 $\mathrm{NaCl}$ 胁迫 $20 \mathrm{~d}$ 时, 两者的 MDA 含量接近, 表明 $\mathrm{NaCl}$ 胁迫下, 'Kerman' 品种的膜脂过氧化程度略低于 '长 果'品种。这些结果表明, $\mathrm{NaCl}$ 胁迫下, “长果”和 'Kerman' 品种的膜系统受到了一定的损伤, 膜脂过 氧化及膜透性对胁迫的响应比较一致, 表现为细胞 膜透性增大, 电解质大量外渗, 膜脂过氧化产物积 累。“长果”和 ‘Kerman’两品种相比较, ‘Kerman’品 种具有较强的耐盐性。

$2.2 \mathrm{NaCl}$ 胁迫对阿月浑子 SOD、POD 和 CAT 活性 的影响

SOD、POD 和 CAT 都是细胞膜系统的保护酶, 在 植物受到盐胁迫时, 对保持体内代谢平衡起着重要 的作用。图 2 显示, 随 $\mathrm{NaCl}$ 浓度的增大, “长果”和 “Kerman” 品种的 SOD、POD 和 CAT 活性均呈现先升 高后下降的趋势, 但达到最大值时的 $\mathrm{NaCl}$ 浓度不 同,而且两个品种保护酶活性的变化也不一致。其 中, “长果”和 “Kerman’ 品种的 SOD 和 POD 活性与 'Kerman' 品种的 CAT 活性在 $\mathrm{NaCl}$ 浓度为 $250 \mathrm{mmol}$ • $\mathrm{L}^{-1}$ 时表现出最高的活性, 而 “长果”品种的 CAT 活 性则在 $\mathrm{NaCl}$ 浓度为 $150 \mathrm{mmol} \cdot \mathrm{L}^{-1}$ 时最高。这表明 在中度 $\mathrm{NaCl}$ 胁迫下, 阿月浑子可以通过提高体内保
护酶活性来维持活性氧代谢平衡, 保持膜系统的稳 定。但在重度 $\mathrm{NaCl}$ 胁迫下, 即 $\mathrm{NaCl}$ 浓度超过 250 $\mathrm{mmol} \cdot \mathrm{L}^{-1}$ 时, 3 种保护酶活性都有所下降。说明阿 月浑子虽然可以耐受一定盐度, 但重度 $\mathrm{NaCl}$ 胁迫超 过了其自身的耐受程度, 植株的细胞膜结构和功能 受到损害, 细胞内自由基不能正常激活保护酶, 致使 保护酶活性下降。随 $\mathrm{NaCl}$ 胁迫时间的延长, $\mathrm{NaCl}$ 胁 迫处理组植株的 SOD、POD 和 CAT 活性变化与对照 组相比, 趋于平缓或基本接近, 表明植株的细胞膜结 构和功能受损害的程度也与 $\mathrm{NaCl}$ 胁迫时间有关。 这可能是随 $\mathrm{NaCl}$ 胁迫处理后时间的延长, 盐分在盆 土中的分布发生了变化, 逐步集中在土壤上层, 从而 减轻了对植株造成的盐害, 保护酶的活性也随盐害 的减弱而降低。

$2.3 \mathrm{NaCl}$ 胁迫下阿月浑子各项生理生化指标间的 相关性分析

$\mathrm{NaCl}$ 胁迫下, “长果”和 ‘ Kerman’ 品种的膜透性 与 MDA 含量都呈极显著正相关 $(p<0.01)$, 即在 MDA 含量增高的同时, 膜透性也表现出增加的趋势 （表 1, 表 2)。“长果”品种的膜透性与 SOD 活性呈正 相关 $(p<0.05)$, MDA 含量与 SOD 和 POD 活性呈显 著正相关 $(p<0.05)$ (表 1$)$ 。而 'Kerman’ 品种的膜 透性和 MDA 含量都与 SOD 活性呈显著正相关 $(p<$ 0.01 ）(表 2)。表明在 $\mathrm{NaCl}$ 胁迫下, 细胞内活性氧代 谢的平衡被破坏, 自由基的大量积累, 造成MDA含

表 $1 \mathrm{NaCl}$ 胁迫下 ‘长果”品种各指标间的相关分析

Table 1 The correlations among the indices of 'Changguo' under $\mathrm{NaCl}$ stress

\begin{tabular}{|c|c|c|c|c|c|}
\hline & $\begin{array}{c}\text { 膜透性 } \\
\text { Membrane permeability }\end{array}$ & $\begin{array}{l}\text { MDA 含量 } \\
\text { MDA content }\end{array}$ & $\begin{array}{l}\text { SOD 活性 } \\
\text { SOD activity }\end{array}$ & $\begin{array}{l}\text { CAT 活性 } \\
\text { CAT activity }\end{array}$ & $\begin{array}{l}\text { POD 活性 } \\
\text { POD activity }\end{array}$ \\
\hline 膜透性 Membrane permeability & 1 & & & & \\
\hline MDA 含量 MDA content & $0.876^{* *}$ & 1 & & & \\
\hline SOD 活性 SOD activity & $0.557^{*}$ & $0.667^{* *}$ & 1 & & \\
\hline CAT 活性 CAT activity & 0.107 & 0.378 & 0.140 & 1 & \\
\hline POD 活性 POD activity & 0.368 & $0.572^{*}$ & $0.742^{* *}$ & $0.521^{*}$ & 1 \\
\hline
\end{tabular}

$* *: p<0.01 \quad *: p<0.05$

表 $2 \mathrm{NaCl}$ 胁迫下 'Kerman' 品种各指标间的相关分析

Table 2 The correlations among the indices of 'Kerman' under $\mathrm{NaCl}$ stress

\begin{tabular}{|c|c|c|c|c|c|}
\hline & $\begin{array}{c}\text { 膜透性 } \\
\text { Membrane permeability }\end{array}$ & $\begin{array}{l}\text { MDA 含量 } \\
\text { MDA content }\end{array}$ & $\begin{array}{l}\text { SOD 活性 } \\
\text { SOD activity }\end{array}$ & $\begin{array}{l}\text { CAT 活性 } \\
\text { CAT activity }\end{array}$ & $\begin{array}{c}\text { POD 活性 } \\
\text { POD activity }\end{array}$ \\
\hline 膜透性 Membrane permeability & 1 & & & & \\
\hline MDA 含量 MDA content & $0.871^{* *}$ & 1 & & & \\
\hline SOD 活性 SOD activity & $0.741^{* *}$ & $0.640^{* *}$ & 1 & & \\
\hline CAT 活性 CAT activity & 0.336 & 0.314 & $0.682^{* *}$ & 1 & \\
\hline POD 活性 POD activity & $0.518^{*}$ & 0.436 & $0.909^{* *}$ & $0.693^{* *}$ & 1 \\
\hline
\end{tabular}



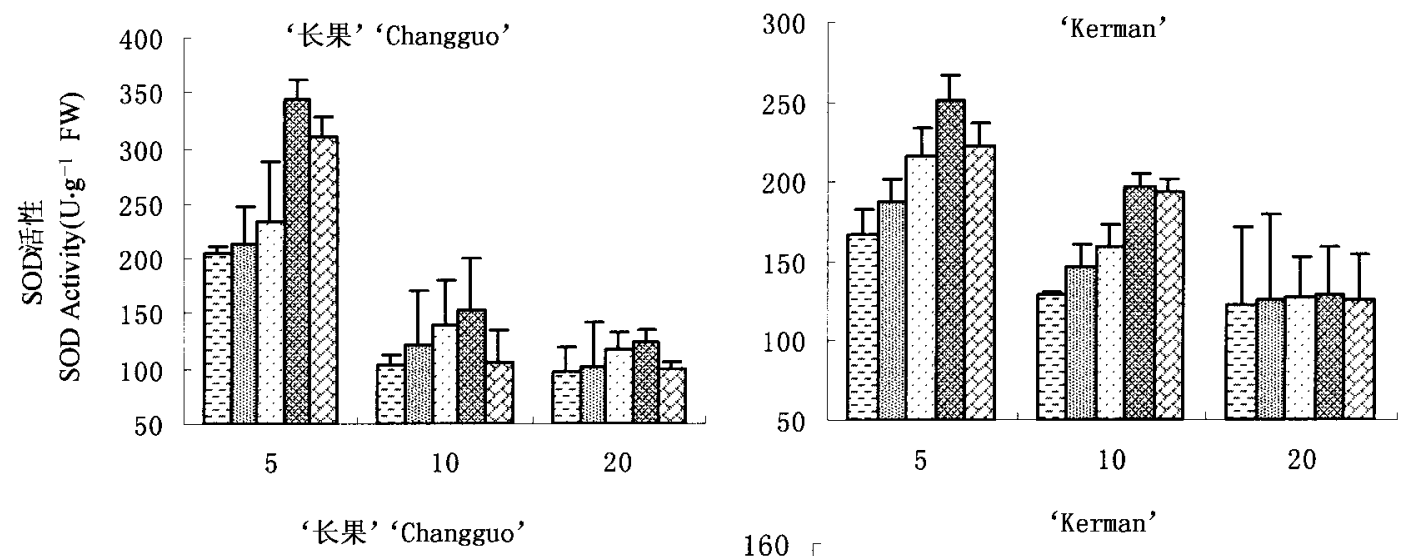
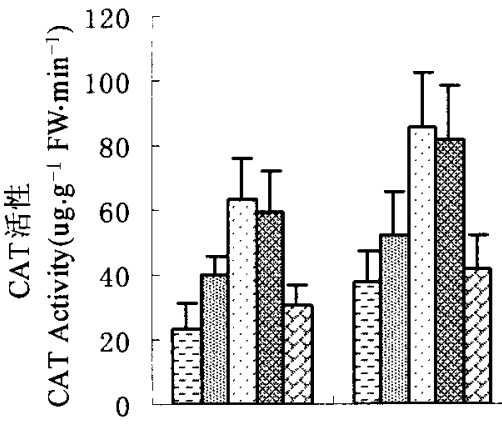

10

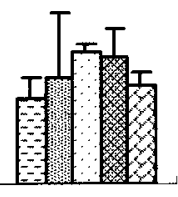

20

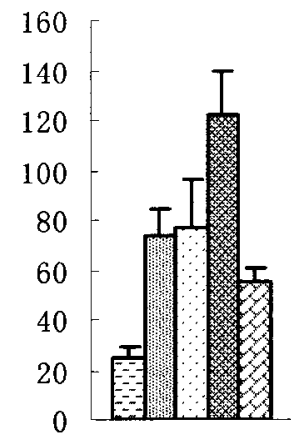

5

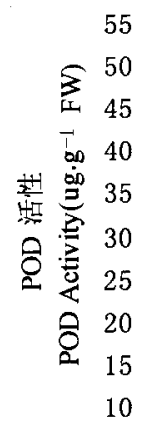

10

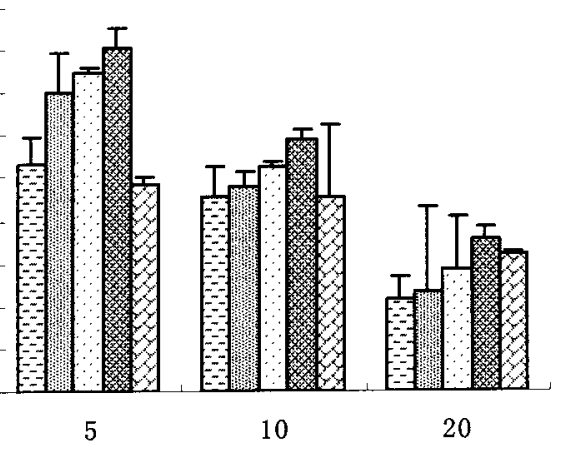

$\mathrm{NaCl}$ 处理天数 $\mathrm{NaCl}$ treatment days (d)

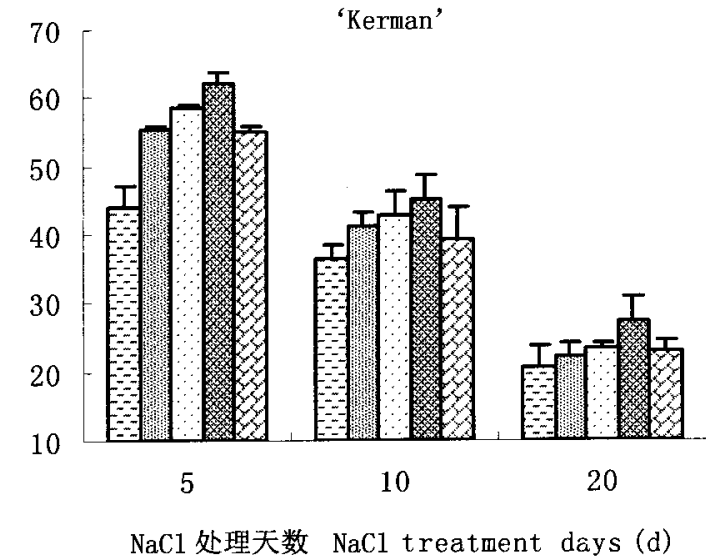

웅 $250 \mathrm{mmol} \cdot \mathrm{L}^{-1} \quad$ 부 $500 \mathrm{mmol} \cdot \mathrm{L}^{-1}$

s $(d)$

\begin{abstract}
口CK 固 $50 \mathrm{mmol} \cdot \mathrm{L}^{-1} \square 150 \mathrm{mmol} \cdot \mathrm{L}^{-1}$
\end{abstract}
'Kerman'

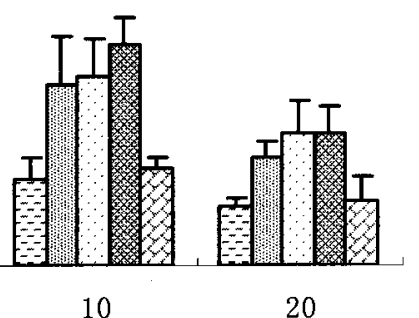

20

图 $2 \mathrm{NaCl}$ 胁迫对阿月浑子 SOD、CAT 和 POD 活性的影响

Fig.2 Effect of $\mathrm{NaCl}$ stress on the activities of SOD, CAT and POD in Pistacia vera

量增高, 细胞膜透性增加, 植物通过维持较高的保护 酶体系 SOD 和 POD 等活性, 从而减弱膜脂过氧化作 用, 缓解了盐分造成的膜伤害。此外, SOD、CAT 和 POD 活性之间也存在一定的相关性, 通常情况下, SOD 活性增加, POD 和 CAT 活性也增加, 表明植物 细胞的膜透性与脂质过氧化产物 (MDA) 含量有关, 也与组织中保护酶活性密切相关。

\section{3 讨论与结论}

膜系统是植物盐害的主要部位。盐胁迫对植物 造成的伤害中, 一个重要的方面是脂质过氧化作用, 而其最终产物之一就是 MDA (Akita \& Cabuslay, 1990)。MDA 作为脂质过氧化作用的产物, 其含量 的多少可代表细胞膜受损伤程度的大小。MDA 通 过影响细胞膜透性及膜蛋白而影响细胞对离子的吸 
收和积累及活性氧代谢系统的平衡, 破坏植物细胞 膜的透性, 影响植物各种生理代谢, 最终影响它的生 长。植物细胞膜透性大小随土壤盐度的变化而变 化。盐胁迫下, 植物细胞的膜透性增加, 耐盐性较强 的植物细胞膜稳定性较强, 膜透性增加较少, 伤害率 低; 而耐盐性弱的植物则相反 (王宝山和姚敦义, 1993)。王洪春 (1981) 认为, 细胞膜结构和功能的紊 乱和膜透性的变化是逆境胁迫作用的关键所在, 由 于渗透胁迫和离子效应使细胞膜系统的完整性受到 破坏, 从而影响细胞内的各种生理代谢过程, 最终对 细胞造成损伤甚至导致死亡。本试验结果表明, $\mathrm{NaCl}$ 胁迫下, “长果” 和 'Kerman' 品种的膜系统均受 到了一定损伤, 胁迫强度越大, 膜系统的损伤也越 大, 其中 'Kerman’ 品种的 MDA 含量增幅较小, 脂质 过氧化程度相对较小, 膜透性随 $\mathrm{NaCl}$ 浓度升高的增 幅也较小, 具有较强的耐盐力。而且随着盐胁迫时 间的延长, 阿月浑子膜透性逐渐下降, 膜系统损伤通 过调节逐渐得到缓解。

植物细胞在正常代谢过程中, 细胞内活性氧的 产生和清除处于动态平衡状态, 活性氧水平很低, 不 会伤害细胞。可是当植物受到胁迫时, 活性氧累积 过多, 这个平衡就被打破。活性氧伤害细胞的机理 在于活性氧化学性质极其活泼, 氧化能力很强, 可与 植物组织中几乎全部生物大分子进行反应, 导致膜 脂过氧化, 保护酶活性下降, 同时还产生较多的膜脂 过氧化产物, 膜的完整性被破坏。其次, 活性氧积累 过多, 也会使膜脂产生脱酯化作用, 磷脂游离, 膜结 构破坏。膜系统的破坏会引起一系列的生理生化紊 乱, 再加上活性氧对一些生物功能分子的直接破坏, 这样植物就会受到伤害, 甚至死亡(王忠, 2000)。但 植物体内同时也存在 ROS 消除系统, 包括 SOD、 CAT、POD、甘露醇、甘氨酸、抗坏血酸和类胡萝卜素 等, 并以此来维持活性氧生成与清除的动态平衡 (Gueta-Dahan et al ., 1997; 陈沁和刘友良, 2000; Hernandez et al., 2000)。盐胁迫下, 植物组织中 ROS 生成大于清除, 使植物膜系统受到伤害, SOD、 CAT 和 POD 活性也发生变化。有研究表明, SOD 的 生理功能主要是清除超氧自由基, 在一定范围内随 盐度的增加 SOD 活性也升高。POD 参与多种生理 代谢, 具有催化多种细胞壁结构成分的合成, 控制细 胞的生长发育, 清除活性氧等作用 (Battacharjee \& Mukherjee, 1996; Dionisio-Sese \& Tobita, 1998)。CAT 能有效地清除生物体内的过氧化氢对细胞的氧化作 用, 保护自身免受・ OH 的毒害。已有许多报道指
出, 当植物处于逆境胁迫时, 抗氧化酶的活性受到影 响, 致使一些活性氧积累, 对细胞膜造成伤害(陈立 松和刘星辉, 2003)。植物在逆境条件下出现的伤害 或植物对逆境的不同抵抗力往往与体内保护酶活性 有关, 而在这些保护酶中, 以 SOD 最为重要。SOD 活性的下降与植物体的衰老是呈正相关的 (Bowler \& von Montagu, 1992; Yu \& Rengel, 1999)。本实验中, 比较这些保护酶相对活性可以看出, $\mathrm{NaCl}$ 胁迫下, “长果”和 “Kerman’ 品种体内 SOD、CAT 和 POD 这 3 种自由基清除酶活性均随盐浓度的升高而增加, 清 除了部分活性氧, 对膜系统起到了一定的保护作用, 其中 SOD 活性相对较高, 在清除活性氧, 防止脂膜 过氧化方面起着主要作用。而且, 'Kerman' 品种体 内的 SOD、CAT 和 POD 活性高于 “长果” 品种, 具有 较强的耐盐力。

$\mathrm{NaCl}$ 胁迫下, 阿月浑子 5 项生理生化指标相关 性分析的结果显示, 部分耐盐指标具有较高的相关 系数, 而其它一些指标之间的相关系数, 尽管未达显 著水平, 但也有一定的规律性。以往的研究表明, MDA 含量的增加与电解质外渗的增大成正相关, 即 认为膜脂过氧化过程是造成膜结构与功能破坏的主 要因素( 陈立松和刘星辉, 2003)。本实验结果与以 前的研究较为吻合。 $\mathrm{NaCl}$ 胁迫下, '长果' 和 ' Kerman’ 品种的膜透性、MDA 含量与 SOD 活性之间的显 著相关性, 进一步表明植物细胞的质膜透性, 与脂质 过氧化产物 (MDA) 含量有关, 也与组织中自由基含 量和保护酶活性密切相关。植物膜系统的稳定性受 到各项指标的影响, 并且这些指标之间不是孤立的, 而是相互联系,密不可分的。一个指标的改变将会 引起其它指标也发生相应的改变。综合各项生理指 标, 可以看出 “长果”和 ‘Kerman’品种均具有一定的 耐盐性,但“Kerman’品种具有相对较强的耐盐力。

$$
\text { 参考 文 献 }
$$

Akita S, Cabuslay GS(1990). Physiological basis of differential response to salinity in rice cultivars. Plant and Soil, 123, 277 294.

Ashworth LJ Jr, Gaona SA, Surber E(1985). Nutritional diseases of pistachio trees: potassium and phosphorus deficiencies and chloride and boron toxicities. Phytopathology, 75, 1084 - 1091 .

Battacharjee S, Mukherjee AK (1996). Ethylene evolution and membrane lipid peroxidation as indicators of salt injury in leaf tissues of amaranths seedlings. Indian Journal of Experimental Biology, 34, $279-281$.

Bowler C, van Montagu M, Inze D (1992). Superoxide dismutase and stress tolerance. Annual Review of Plant Physiology and 
Plant Molecule Biology, 43, 83 - 116.

Chen LS (陈立松), Liu XH(刘星辉) (2003). Fruit Tree AntiStress Physiology (果树逆境生理). China Agriculture Press, Beijing, 16-256. (in Chinese)

Chen Q(陈沁), Liu YL(刘友良) (2000). Effect of glutathione on active oxygen scavenging system in leaves of barley seedlings under salt stress. Acta Agronomica Sinica (作物学报), 26, $365-$ 371. (in Chinese with English abstract)

Dionisio-Sese ML, Tobita S(1998). Antioxidant responses of rice seedlings to salinity stress. Plant Science, 135, 1 -9.

Editorial Committee of Flora of China, Chinese Academy of Sciences (中国科学院中国植物志编辑委员会) (1980). Flora of China (Vol.45, No. 1) (中国植物志 45 卷第一期). Science Press, Beijing, 94 - 96. (in Chinese)

Gueta-Dahan Y, Yaniv Z, Zilinskas BA, Ben-Hayyim G (1997). Salt and oxidative stress: similar and specific responses and their relation to salt tolerance in citrus. Planta, 203, 460-469.

Hernandez JA, Jimenez A, Mullineaux P, Sevilla F (2000). Tolerance of pea (Pisum sativum L. ) to long term salt stress is associated with induction of antioxidant defenses. Plant Cell Biology, $23,853-862$.

Li HS(李合生) (2000). The Principle and Experimental Technology of Plant Physiology and Biochemistry (植物生理生化实验原 理和技术). Higher Education Press, Beijing, 164 - 169. (in Chinese)

Lin QF (林栖风), Li GY (李冠一) (2000). The status and advances of plant salt-tolerance. Bioengineering Evolvement (生物 工程进展), 20(2), 20-25. (in Chinese with English abstract) Pan RC (潘瑞炽), Dong YD (董愚得) (1995). Plant Physiolo$g y$ (植物生理学) 3rd edn. Higher Education Press, Beijing, 318 - 335. (in Chinese)

Picchioni GA, Miyamota S(1990). Salt effects on growth and ion uptake of pistachio rootstock seedlings. Journal of the American Society for Horticultural Science, 115, 647 - 653 .

Sepskhah AR, Maftoin M(1988). Relative salt tolerance of pistachio cultivars. Horticulture Science, 63, 157 - 162 .

Wang BS(王宝山), Yao DY (姚敦义) (1993). The effect of salt tolerance on membrane permeability, lipid peroxidization and SOD activity of callus in Elacagnus angustifolia. Journal of Hebei Agricultural University (河北农业大学学报), 16(3), 20-24. (in Chinese)

Wang HC (王洪春) (1981). Plant adaptation to stresses. Plant Physiology Communications (植物生理学通讯), 6, 72 - 73 . (in Chinese)

Wang JH(王继和), Yang ZH(杨自辉) (2001). Research of the comprehensive technologies on transformation and utilization of saline land in arid areas. Chinese Journal of Eco-Agriculture (中 国生态农业学报), 9, 64-66. (in Chinese with English abstract)

Wang Z(王忠) (2000). Plant Physiology (植物生理学). China Agriculture Press, Beijing, 422 - 439. (in Chinese)

Yu Z, Rengel Q (1999). Drought and salinity differentially influence activities of superoxide dismutase in narrow leafed lupines. Plant Science, 141, 1 - 11 .

Zhang KC(张开春) (2000). The Handbook of Fruit Tree Breeding (果树育苗手册). China Agriculture Press, Beijing, 302 304. (in Chinese)

Zhao SJ(赵氏杰), Xu CC(许长成), Zou Q(邹琦), Meng QW (孟庆伟) (1994). Improvements of method for measurement of malondialdehyde in plant tissues. Plant Physiology Communications (植物生理学通讯), 30, 207-210. (in Chinese)

Zhu GL(朱广廉), Zhong HW (钟海文), Zhang AQ (张爱琴) (1990). Experiments of Plant Physiology (植物生理学实验). Peking University Press, Beijing, 252 - 254. (in Chinese)

责任编委: 李银心 责任编辑: 刘丽娟 